preparing all these X-ray treatments, and as a physicist, it struck me as a very rough process," Dr Cormack said. "How can you know how to treat something in the middle of the head if you do not have proper information of the effect on the radiation of the material through which it has to pass?"

The question appealed to him as a scientist. But the task of working out the answer was "mostly a mathematical one", extrapolating what physicists already knew about the attenuation of radiation passing through a homogenous material to similar calculations for inhomogenous materials.

"After publication in 1963 there was virtually no response at all, except for a few physicians, until someone came along in about 1970 to say that similar ideas had been developed by engineers at EMI,"' says Mr Cormack. He says that he has never met Godfrey Hounsfield, with whom he now shares the Nobel Prize, but adds that "I have heard that the is an inventive person."

Cormack's lack of involvement with the commercial development of computerised comography (CT) scanners means that he has been able to watch from the sidelines the intense debate which has taken place in the US - and which promises to be revived by the Nobel Prize award - over the use of the technology. In particular, this debate has focussed on the extent to which the federal government should support the growth of a technology whose extremely high capital costs have contributed to the rapid increase in hospital care costs in recent years.

$\mathrm{Mr}$ Cormack is critical of the US government's decision to place stringent restrictions on the use of CT. This move followed a report from the National Academy of Science's Institute of Medicine pointing out that in many cases, conventional X-ray techniques were much cheaper and might be just as effective (Nature, 12 May 1977).

"The arguments against the CT. scanner leave out a lot of things. In doing a cost-benefit analysis, for example, how much to you put in for the pain and suffering of patients for whom exploratory surgery might be avoided by using the scanner? ' Mr Cormack says.

"I think the scanner has become a symbol, it is used as a whipping boy as a way of pointing out the expense of medical care; intensive care units probably cost a lot more money, but you do not usually hear about them."

At the same time, however, he admits that the cost of the basic equipment might be brought down if manufacturers were not under great pressure from physicians to improve its performance, for example through faster scans or higher resolution. "A substantial part of the costs must still be in development. If they could standardise the model, the costs would probably go down a lot," Mr Cormack says.

David Dickson

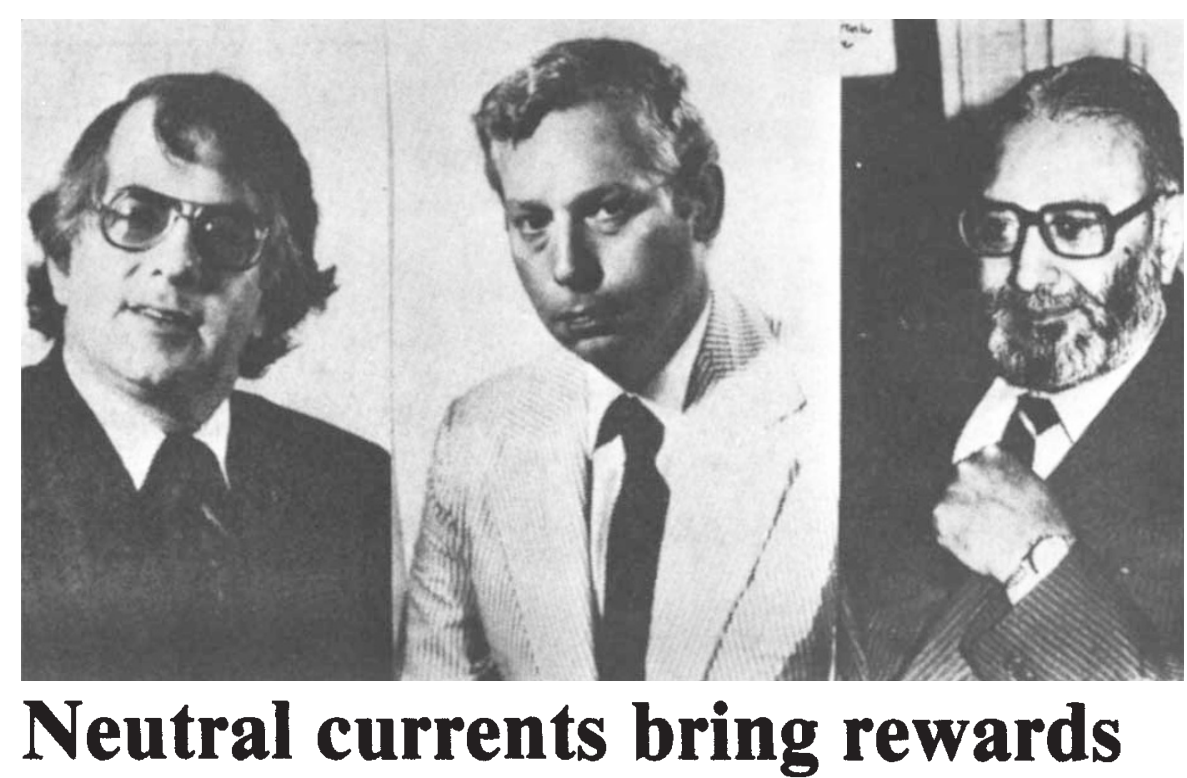

THIS is the prize that many physicists have been waiting for - not least Abdus Salam, of Imperial College, London, and the International Centre for Theoretical Physics, Trieste, and Steven Weinberg of Harvard, whose unified model of the action of the weak and electromagnetic forces has been finding corroboration in an increasing number of experiments.

But one of the severest tests of the theory - the discovery of heavy counterparts to the photon, the 'intermediate vector bosons', is yet to come, and a few will no doubt consider the prize to be premature. Experiments planned at CERN, the European centre for subnuclear physics, for 1982 are expected to provide the first direct evidence of the particles.

However the citation refers to the prediction "inter alia" of neutral currents, and here the theory is on firm ground. Sheldon Glashow, of MIT, played a key role in their prediction independently of the machinery of the Salam-Weinberg model, and hence his well deserved share in the prize.

The term 'neutral current' is a somewhat confusing epithet for weak interactions not involving the exchange of electric charge. For example, the familiar weak decay of atomic nuclei involves neutrons changing into protons (or vice versa), the excess charge being taken up by the creation of an electron and an antineutrino (or their antiparticles). This is supposed to take place by the emission and reabsorption (exchange) of a charged intermediate vector boson between the nucleon and electron systems. Neutral current interactions, on the other hand, involve the exchange of a neutral IVB, and so change protons into protons, electrons into electrons, and so on.

The weak force being extremely weak, the effects of these "neutral' exchanges are completely masked by the analagous exchange of photons which creates the electromagnetic force between, say, protons and electrons. (It is this analogy, indeed, which allows the two forces to be unified in an all embracing theory.)

The detection of neutral currents has thus proved extremely difficult, and its history has not been without its conflicts of evidence. The first observation was made with neutrinos (where electromagnetic effects are absent) at CERN; others followed, and gradually the neutrino evidence has built up into an impressive body of evidence not only for the existence of the neutral current, but for the exact form of it predicted by Glashow and incorporated by Salam and Weinberg in their (independently generated) 1967 and 1968 unified theory. More recently, a brilliant experiment with polarised electrons at the Stanford Linear Accelerator Center in California measured the interference between the electromagnetic and neutral weak interactions confirmed predictions.

Robert Walgate

\section{Boron and phosphorus}

Herbert C Brown of Purdue University, Indiana, and Georg Wittig of Heidelberg University share the Nobel Prize for chemistry. The citation describes their work on the application of boron and phosphorus compounds respectively to synthetic organic chemistry.

Professor Wittig, who is now over 80 but still academically active, is best known for the development of Wittig reagents, a class of phosphorus-based compounds used to form carbon-carbon double bonds at precisely predetermined places in organic molecules. They have applications in the synthesis of many compounds, including prostaglandins.

Professor Brown, on the other hand, has developed a series of very versatile organoboron compounds which can selectively modify functional groups in organic molecules, and is also renowned for his theoretical contributions to carbo-cation chemistry. His birthplace was in the UK, buthe haspursued hiscareer in the US. between electrons and protons, and also 